\title{
The Usefulness of the Kurashiki Prehospital Stroke Scale in Identifying Thrombolytic Candidates in Acute Ischemic Stroke
}

\author{
Jieun Jang, ${ }^{1}$ Sung Phil Chung, Incheol Park, Je Sung You ${ }^{1,2}$ Hye Sun Lee, ${ }^{3}$ Jong Woo Park, \\ Tae Nyoung Chung, ${ }^{5}$ Hyun Soo Chung, ${ }^{1}$ and Hahn Shick Lee ${ }^{1}$ \\ 'Department of Emergency Medicine, Yonsei University College of Medicine, Seoul; \\ 2Department of Emergency Medicine, Graduate School of Medicine, Kangwon National University, Chuncheon; \\ ${ }^{3}$ Department of Research Affairs, Biostatistics Collaboration Unit, Yonsei University College of Medicine, Seoul; \\ ${ }^{4}$ Department of Emergency Medicine, Changwon Fatima Hospital, Changwon; \\ ${ }^{5}$ Department of Emergency Medicine, Bundang CHA Hospital, CHA University College of Medicine, Seongnam, Korea.
}

\author{
Received: May 7, 2013 \\ Revised: June 24, 2013 \\ Accepted: July 9, 2013 \\ Corresponding author: Dr. Je Sung You, \\ Department of Emergency Medicine, \\ Gangnam Severance Hospital, \\ Yonsei University College of Medicine, \\ 211 Eonju-ro, Gangnam-gu, \\ Seoul 135-720, Korea. \\ Tel: 82-2-2019-3030, Fax: 82-2-2019-4820 \\ E-mail: youjsmd@yuhs.ac
}

- The authors have no financial conflicts of interest.

\section{(c) Copyright:}

Yonsei University College of Medicine 2014

This is an Open Access article distributed under the terms of the Creative Commons Attribution NonCommercial License (http://creativecommons.org/ licenses/by-nc/3.0) which permits unrestricted noncommercial use, distribution, and reproduction in any medium, provided the original work is properly cited.
Purpose: The severity of a stroke cannot be described by widely used prehospital stroke scales. We investigated the usefulness of the Kurashiki Prehospital Stroke Scale (KPSS) for assessing the severity of stroke, compared to the National Institutes of Health Stroke Scale (NIHSS), in candidate patients for intravenous or intraarterial thrombolysis who arrived at the hospital within 6 hours of symptom onset. Materials and Methods: We retrospectively analyzed a prospective registry database of consecutive patients included in the Emergency Stroke Therapy program. In the emergency department, the KPSS was assessed by emergency medical technicians. A cutoff KPSS score was estimated for candidates of thrombolysis by comparing KPSS and NIHSS scores, as well as for patients who actually received thrombolytic therapy. Clinical outcomes were compared between patients around the estimated cut-off. The independent predictors of outcomes were determined using multivariate logistic regression analysis. Results: Excellent correlations were demonstrated between KPSS and NIHSS within 6 hours $(\mathrm{R}=0.869)$ and 3 hours $(\mathrm{R}=0.879)$ of hospital admission. The optimal threshold value was a score of 3 on the KPSS in patients within 3 hours and 6 hours by Youden's methods. Significant associations with a KPSS score $\geq 3$ were revealed for actual intravenous administration of tissue plasminogen activator (IV-tPA) usage [odds ratio (OR) 125.598; 95\% confidence interval (CI) 16.443-959.368, $p<0.0001]$ and actual IV-tPA or intra-arterial urokinase (IA-UK) usage (OR 58.733; 95\% CI 17.272-199.721, $p<0.0001$ ). Conclusion: The KPSS is an effective prehospital stroke scale for identifying candidates for IV-tPA and IA-UK, as indicated by excellent correlation with the NIHSS, in the assessment of stroke severity in acute ischemic stroke.

Key Words: Prehospital emergency care, stroke, thrombolytic therapy

\section{INTRODUCTION}

Stroke is one important cause of mortality and morbidity as well as socioeconomic 
burdens. ${ }^{1}$ The ultimate goal of stroke care is to minimize acute brain injury and maximize patient recovery. ${ }^{2} \mathrm{~A}$ common concept is that "time is brain" due to the time-sensitive nature of brain tissue injury. ${ }^{3}$ A community-oriented "stroke chain of survival," much like the adult chain of survival for sudden cardiac arrest, includes rapid recognition of stroke warning signs and activation of the emergency response system: rapid emergency medical system (EMS) dispatch, transport, and prehospital notification; triage to a stroke center; and finally, rapid diagnosis, treatment, and disposition in the hospital. ${ }^{2}$

EMS providers can identify stroke patients with prehospital stroke screen tools in 30-60 seconds using the Cincinnati Prehospital Stroke Scale (CPSS) or the Los Angeles Prehospital Stroke Screen (LAPSS). ${ }^{4-6}$ However, LAPSS and CPSS have only been used to discriminate between acute stroke and urgent neurologic disorders. ${ }^{5,6}$

In stroke treatment, intravenous administration of tissue plasminogen activator (IV-tPA) can improve outcomes for a broad spectrum of patients able to be treated within 3 hours of symptom onset. ${ }^{4}$ In addition to IV-tPA for brain salvage, intra-arterial thrombolysis can benefit select patients who have had a major stroke within 6 hours of symptom onset, but who are not candidates for IV-tPA. ${ }^{7}$ EMS personnel should consider triage to a stroke center for greater stroke management, and a rapid triage system for assessing stroke severity should be used in the emergency department (ED) to minimize diagnostic and therapeutic delay. ${ }^{2}$

The National Institutes of Health Stroke Scale (NIHSS) provides important information about the severity of a stroke and informs early prognosis and decisions about acute treatment. ${ }^{4}$ However, this stroke-scoring scale is relatively timeconsuming and unnecessarily sophisticated for field use. ${ }^{8} \mathrm{Al}-$ though CPSS and LAPSS can help rapidly recognize the symptoms of acute stroke, important information about the severity of stroke cannot be determined by these stroke scales. $^{3}$

The Kurashiki Prehospital Stroke Scale (KPSS) was developed to assist in deciding whether to transfer a patient to an appropriate EMS stroke facility in Japan. ${ }^{9}$ Kimura, et al. ${ }^{9}$ revealed concordance in stroke severity between the KPSS and NIHSS in assessing acute stroke and transient ischemic attack (TIA) for patients admitted within 3 hours of symptom onset. Additionally, early administration of intra-arterial thrombolysis should be considered as a possible measure to improve clinical outcomes both in the field and the ED. ${ }^{4}$ However, intra-arterial thrombolysis requires a higher capa- bility of stroke management compared to simple usage of IV-tPA.

Herein, we investigated the usefulness of KPSS in assessing the severity of stroke compared to the NIHSS in patients who may be candidates for thrombolysis who arrived to a hospital within 6 hours of symptom onset.

\section{MATERIALS AND METHODS}

We retrospectively analyzed a prospective registry database of all consecutive patients included in the Brain Salvage through Emergency Stroke Therapy (BEST) program conducted at a 2000-bed tertiary academic hospital with an annual ED census of 65000 . The institutional review board of Yonsei University Health System reviewed and approved the study. The study included patients with ischemic stroke or TIA on ED arrival within 6 hours of symptom onset between Sep 1, 2010 and Sep 30, 2011.

In our hospital, the BEST program, based on the computerized physician order entry (CPOE) system, was used to effectively guide the operation of a pre-existing stroke team since 2004. The CPOE system was used for program activation/deactivation, communication/consultation processes, entering predetermined standing order sets, providing specific protocols/guidelines, and assessment of the program efficacy. ${ }^{7}, 10$

Upon arrival of a patient to the ED, physicians, nurses, or emergency medical technicians in the triage area identified candidates for the BEST program as soon as possible according to predetermined guidelines. The guidelines included the five stroke warning signs (sudden numbness or weakness of the face, arms, or legs; sudden confusion, trouble speaking or understanding speech; sudden trouble seeing in one or both eyes; sudden severe headache with no known cause; sudden trouble walking, dizziness, loss of balance, or loss of coordination) prepared by the American Heart Association (AHA) stroke council for the general population. ${ }^{47,10}$ When a patient had at least one warning sign and arrived within 12 hours after symptom onset, an ED physician in the triage area activated the BEST program by selecting the activation icon at the order entry window. Once the program was activated, the patient's name in the patient list was highlighted orange; in this way, every team member could easily and immediately recognize BEST patients. ${ }^{71}$ At the same time, an ED physician immediately consulted with an on-call neurologist and senior ED physicians. To 
effectively inform computed tomography (CT) and blood test technicians, medical orders for a CT scan and blood testing automatically activated an alarm, a beeping sound, and a pop-up window on the technician's monitor. ${ }^{710}$ Thus, at the same time medical orders were submitted, technicians were alerted and could prepare for the arrival of the patient or blood samples, enabling immediate and rapid examinations. The BEST program was deactivated either after IV-tPA administration to the patient or once it was determined that thrombolytic treatment would not be indicated. ${ }^{7,10}$

When a patient suspected of suffering a stroke arrived in the ED, the BEST program was activated according to a predetermined protocol. Once admitted to the ED, the KPSS was assessed by one of six emergency medical technicians (EMTs) included in the study, while the NIHSS score was determined by a neurologist in the ED. Patients were excluded from the study if symptom onset occurred more than 6 hours before admission, if a hemorrhagic stroke or a brain tumor was revealed in non-contrast CT, or if another medical disease such as encephalitis, seizure, hypoglycemia, or syncope was diagnosed after admission. To achieve a precise diagnosis, we performed diffusion-weighted magnetic resonance imaging and angiography or brain $\mathrm{CT}$ angiography after non-contrast CT. According to previously published criteria, IV-tPA was administered to patients diagnosed with acute stroke of less than 3 hours duration, and intra-arterial thrombolysis was performed in selected patients with a major stroke of less than 6 hours duration occurring by middle cerebral artery, vertebral, or basilar artery occlusion and who were not otherwise candidates for IVtPA or for whom there was no improvement after administration of IV-tPA., 2,4

\section{Statistics}

Demographic and clinical data are presented as medians (q1, q3) or a proportion, as appropriate. Correlation between KPSS and NIHSS scores was analyzed using Spearman's rank correlation. Patients with a NIHSS score of 5-22 were considered candidates for IV-tPA if symptom onset was less than 3 hours before admission. A NIHSS score $>5$ qualified patients for intra-arterial urokinase (IA-UK) treatment if symptom onset had occurred less than 6 hours previous.

A cut-off KPSS was estimated using Youden's method among candidates for IV-tPA and IA-UK by comparing KPSS and NIHSS. Youden's method was used to find an optimal cutoff point in an receiver operating characteristic (ROC) curve to maximize sensitivity and specificity.
We compared baseline characteristics by Mann-Whitney test and chi-square test. The independent predictors of outcomes were determined using multivariate logistic regression analysis. For this analysis, variables of $p<0.05$ on the univariate analysis were entered. All values of $p<0.05$ were considered significant. Statistical analysis was conducted using SAS version 9.2 (SAS Institute Inc., Cary, NC, USA).

\section{RESULTS}

A total of 737 consecutive patients were admitted to our ED and included in the BEST program. A total of 191 patients with symptom onset of greater than 6 hours previous were excluded. A total of 254 patients diagnosed with either hemorrhagic stroke ( $n=93)$, brain tumors $(n=3)$ or with another medical disease $(\mathrm{n}=155)$ were also excluded.

A total of $295(40.0 \%)$ consecutive patients diagnosed with ischemic stroke or TIA and who presented within 6 hours after symptom onset were included in this study. Of these, 11 were excluded due to incomplete data such as incomplete stroke scoring, lack of evaluation for stroke due to combined life-threatening diseases, or transfer from another hospital after magnetic resonance or IV-tPA. One hundred eighty-four of the remaining 284 patients (64.8\%) were admitted within 3 hours. In this study, IV-tPA was administered to 41 patients (22.3\%), IA-UK was administered to five patients $(2.7 \%)$, and sequential combination t-PA and IA-UK was administered to 11 patients (6.0\%) (Fig. 1).

Fig. 2 shows a scatter diagram of KPSS scores determined by EMT and NIHSS scores by neurologists for patients with onset within 6 hours (Fig. 2A) and 3 hours (Fig. 2B) before admission. Excellent correlation was demonstrated between KPSS and NIHSS scores within 6 hours $[\mathrm{R}=0.869 ; 95 \%$ confidence interval (CI, 0.837-0.895), $p<0.0001]$ and 3 hours $[\mathrm{R}=0.879 ; 95 \% \mathrm{CI}(0.840-0.907), p<0.0001]$ before admission (Fig. 2).

Table 1, an ROC curve, which measured the effectiveness of KPSS as a diagnostic scoring tool for stroke severity by comparing KPSS and NIHSS, indicated an optimal threshold value at the cut-off score of 3 on the KPSS in patients within 3 hours and 6 hours. For actual usage of IVtPA and IA-UK, the cut-off value of 3 provided $98.1 \%$ sensitivity and $70.7 \%$ specificity for administration of IV-tPA within 3 hours and $95.5 \%$ sensitivity and $74.3 \%$ specificity for administration of IV-tPA or IA-UK within 6 hours. There were significant differences in KPSS cutoffs $<3$ and $\geq 3$ for 


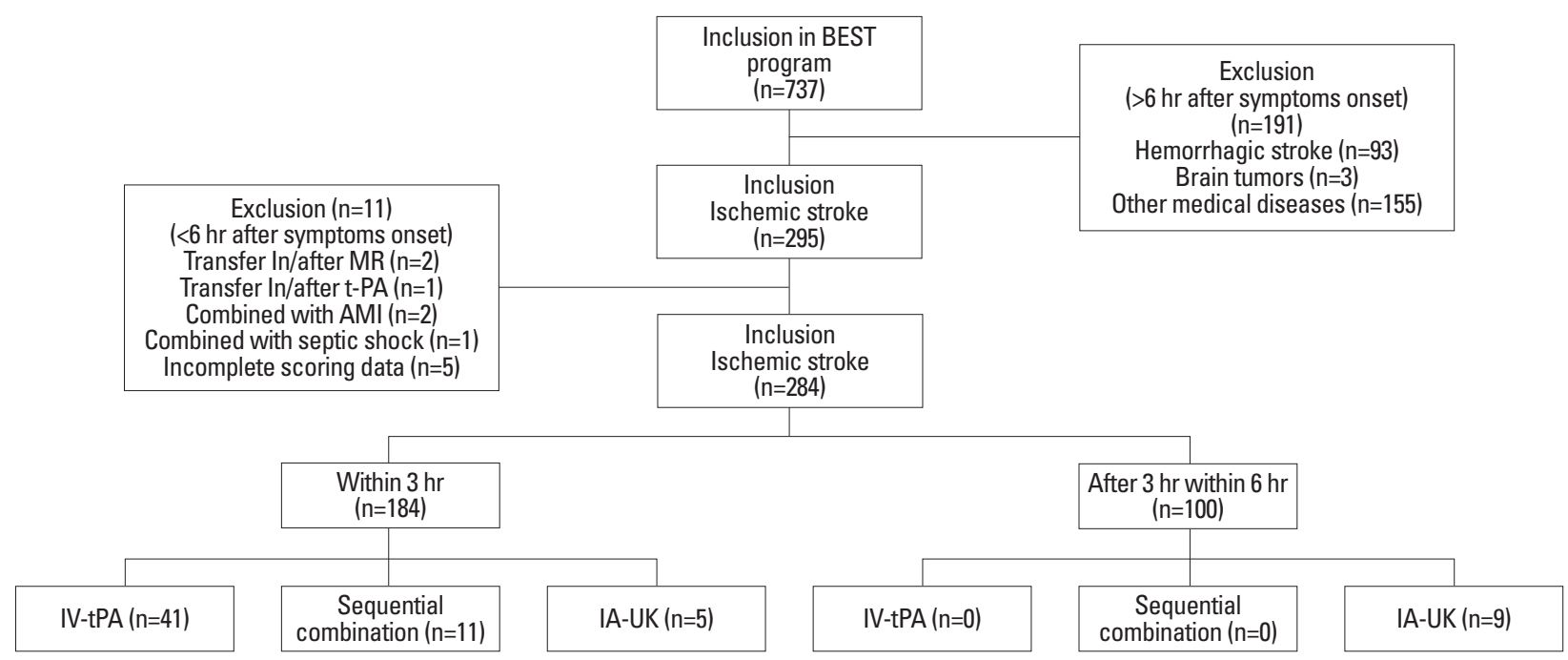

Fig. 1. Enrollment and clinical outcomes. BEST, Brain Salvage through Emergency Stroke Therapy; IV-tPA, intravenous administration of tissue plasminogen activator; IA-UK, intra-arterial urokinase; MR, magnetic resonance; AMI, acute myocardial infarction.
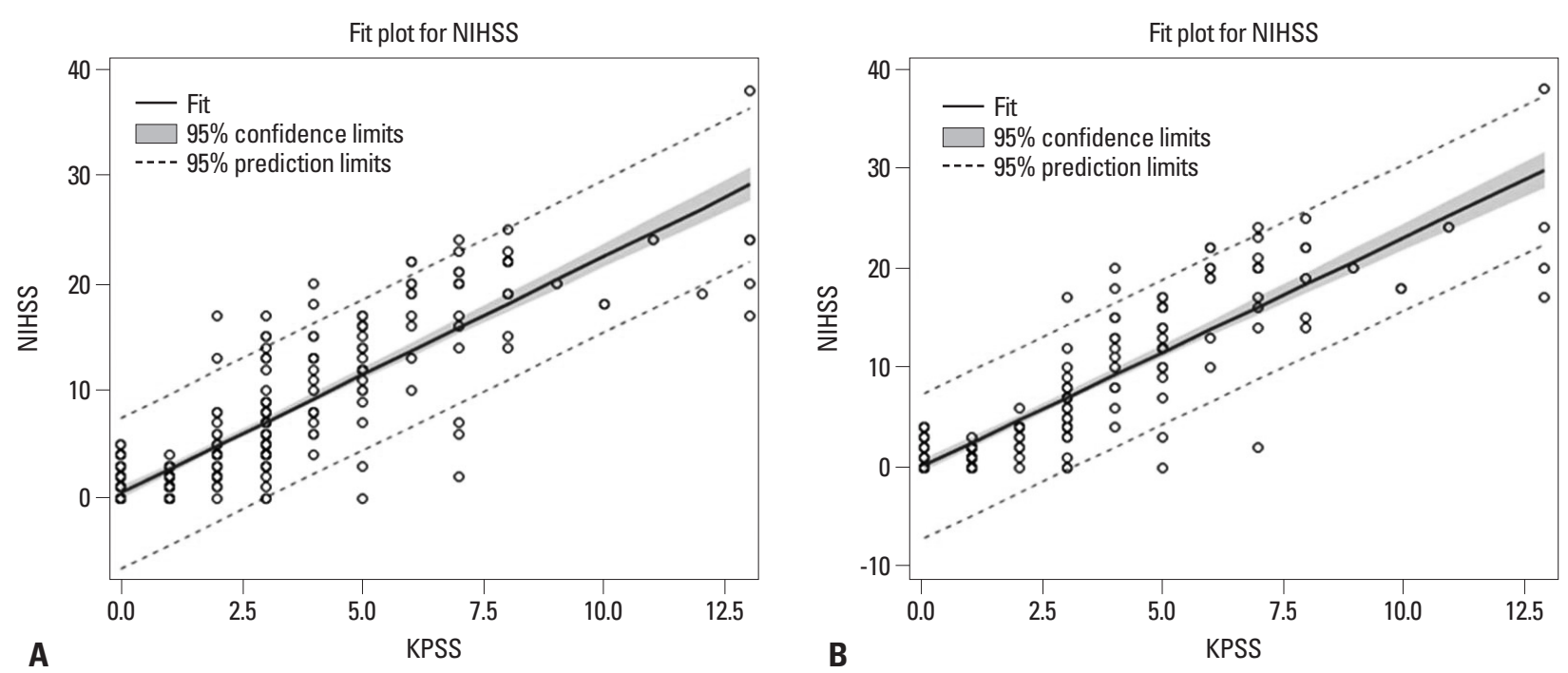

Fig. 2. An excellent correlation was demonstrated between KPSS and NIHSS within 6 hours (A) $[\mathrm{R}=0.869 ; 95 \% \mathrm{Cl}(0.837-0.895)$, $p<0.0001]$ and 3 hours (B) $[\mathrm{R}=0.879 ; 95 \% \mathrm{Cl}(0.840-0.907), p<0.0001]$ before admission. KPSS, Kurashiki Prehospital Stroke Scale; NIHSS, National Institutes of Health Stroke Scale.

actual usage of IV-tPA $(p<0.0001)$ or IA-UK $(p<0.0001)$ (Table 2). Significant associations for KPSS scores $\geq 3$ were revealed in actual IV-tPA usage [odds ratio (OR) 125.598; 95\% CI 16.443-959.368, $p<0.0001]$ and actual IV-tPA or IAUK usage (OR 58.733; 95\% CI 17.272-199.721, $p<0.0001$ ) (Table 3).

\section{DISCUSSION}

The KPSS was developed in 2008 to assess the severity of a stroke. This scoring system was constructed using four metrics including level of consciousness, disturbance of consciousness, motor weakness, and language, with total scores ranging from 0 to 13 . The maximum of 13 points represented the most severe symptoms (Fig. 3). Kimura, et al. ${ }^{9}$ revealed that the KPSS can be used to identify stroke patients and to evaluate stroke severity. Additionally, Iguchi, et al. ${ }^{3}$ also demonstrated that KPSS is in reliable concordance with NIHSS for patients within 3 hours of stroke onset, but that patients with a KPSS $\geq 4$ had a relatively greater chance of receiving IV-tPA. IV-tPA is currently the only FDA-approved medical therapy for patients with acute ischemic stroke. ${ }^{4}$ Nevertheless, several recent studies have demonstrated that intra-arterial thrombolysis may be beneficial in the treatment of carefully selected patients with middle cerebral, vertebral, or basilar artery occlusion or occlusion of the anterior circulation. ${ }^{4,11}$ 
Table 1. A Cut-Off KPSS Value Was Estimated Using Youden's Method for IV-tPA and IA-UK Candidates by Comparing between KPSS and NIHSS and by Estimating Cut-Off Values for Patients Who Actually Received IV-tPA and IA-UK in the Emergency Department

\begin{tabular}{lccccc}
\hline & Cut-off value & Sensitivity & Specificity & AUC & Youden's \\
\hline $\begin{array}{l}\text { Actual IV-tPA usage by KPSS } \\
\text { within } 3 \text { hrs }\end{array}$ & 3 & $98.1 \%[94.3,100.0]$ & $70.7 \%[64.8,76.5]$ & $0.844[0.809,0.879]$ & 0.688 \\
$\begin{array}{l}\text { Actual IV-tPA or IA-UK usage } \\
\text { by KPSS within 6 hrs }\end{array}$ & 3 & $95.5 \%[90.4,100.0]$ & $74.3 \%[68.5,80.1]$ & $0.849[0.810-0.887]$ & 0.698 \\
$\begin{array}{l}\text { Comparison between KPSS and } \\
\text { NIHSS (5-22) within 3 hrs }\end{array}$ & 3 & $89.4 \%[83.5,95.3]$ & $85.6 \%[80.4,90.7]$ & $0.875[0.836,0.914]$ & 0.750 \\
$\begin{array}{l}\text { Comparison between KPSS and } \\
\text { NIHSS (5-maximum) within 6 hrs }\end{array}$ & 3 & $90.1 \%[84.5,95.6]$ & $89.0 \%[84.4,93.7]$ & $0.896[0.859,0.932]$ & 0.791 \\
\hline
\end{tabular}

KPSS, Kurashiki Prehospital Stroke Scale; NIHSS, National Institutes of Health Stroke Scale; IV-tPA, intravenous administration of tissue plasminogen activator; IA-UK, intra-arterial urokinase; AUC, area under curve.

Youden's index: (sensitivity+specificity-1).

Table 2. Clinical Outcomes of Patients with Ischemic Stroke Using a KPSS Cut-Off of 3

\begin{tabular}{|c|c|c|c|}
\hline & KPSS <3 (n=165) & $\operatorname{KPSS} \geq 3(n=119)$ & $p$ value \\
\hline \multicolumn{4}{|l|}{ Demographics } \\
\hline Age & $66.0[55.0,73.0]$ & $72.0[63.0,77.0]$ & $<0.0001$ \\
\hline Male $(\%)$ & $103(62.4)$ & $64(53.8)$ & 0.1443 \\
\hline Onset to door time (min) & $126.0[64.0,226.0]$ & $76.0[41.0,183.0]$ & 0.0004 \\
\hline Coronary artery disease (\%) & $26(15.8)$ & $20(16.8)$ & 0.8128 \\
\hline Hypertension $(\%)$ & $111(67.3)$ & $82(68.9)$ & 0.7708 \\
\hline Diabetic mellitus (\%) & $38(23.0)$ & $33(27.7)$ & 0.3667 \\
\hline Hypercholesterolemia (\%) & $23(13.9)$ & $10(8.4)$ & 0.1509 \\
\hline Smoking (\%) & $73(44.2)$ & $43(36.1)$ & 0.1702 \\
\hline Atrial fibrillation (\%) & $23(13.9)$ & $37(31.1)$ & 0.0005 \\
\hline Old cerebrovascular disease $(\%)$ & $50(30.3)$ & $35(29.4)$ & 0.8717 \\
\hline \multicolumn{4}{|l|}{ Clinical Outcome (\%) } \\
\hline Stroke type & & & $<0.0001$ \\
\hline Ischemic stroke & $101(61.2)$ & $112(94.1)$ & \\
\hline Transient ischemic attack & $64(38.8)$ & $7(5.9)$ & \\
\hline \multicolumn{4}{|l|}{ Actual usage (\%) } \\
\hline IV-tPA & $1(0.6)$ & $51(42.9)$ & $<0.0001$ \\
\hline IA-UK & $2(1.2)$ & $23(19.3)$ & $<0.0001$ \\
\hline IV-tPA or IA-UK & $3(1.8)$ & $63(52.9)$ & $<0.0001$ \\
\hline Median NIHSS (\%) & $1.0[0.0,2.0]$ & $13.0[7.0,18.0]$ & $<0.0001$ \\
\hline NIHSS (5-22) & $11(6.7)$ & $93(78.2)$ & $<0.0001$ \\
\hline NIHSS (5-maximum) & $11(6.7)$ & $100(84.0)$ & $<0.0001$ \\
\hline
\end{tabular}

NIHSS, National Institutes of Health Stroke Scale; KPSS, Kurashiki Prehospital Stroke Scale; IV-tPA, intravenous administration of tissue plasminogen activator; IA-UK, intra-arterial urokinase.

[]: interquartile range.

Table 3. The Independent Predictors of Outcome Were Determined Using Multivariate Logistic Regression Analysis

\begin{tabular}{|c|c|c|c|c|}
\hline & \multicolumn{2}{|c|}{ Actual IV-tPA usage } & \multicolumn{2}{|c|}{ Actual IV-tPA or IA-UK usage } \\
\hline & OR $(95 \% \mathrm{CI})$ & $p$ value & OR $(95 \% \mathrm{CI})$ & $p$ value \\
\hline Age & - & - & - & - \\
\hline Onset to Door time (min) & $0.980[0.972-0.988]$ & $<0.0001$ & $0.990[0.985-0.994]$ & $<0.0001$ \\
\hline Hypercholesterolemia & $0.120[0.012-1.156]$ & 0.0665 & $0.189[0.036-0.982]$ & 0.0476 \\
\hline Atrial fibrillation & - & - & - & - \\
\hline $\mathrm{KPSS} \geq 3$ & $125.598[16.443-959.368]$ & $<0.0001$ & $58.733[17.272-199.721]$ & $<0.0001$ \\
\hline
\end{tabular}

KPSS, Kurashiki Prehospital Stroke Scale; IV-tPA, intravenous administration of tissue plasminogen activator; IA-UK, intra-arterial urokinase; OR, odds ratio; $\mathrm{Cl}$, confidence interval. 


\begin{tabular}{|c|c|c|c|}
\hline & & \multicolumn{2}{|c|}{ Scoring (total disorder: 13 points) } \\
\hline \multirow{4}{*}{ Consciousness level } & & \multirow{2}{*}{\multicolumn{2}{|c|}{ Normal: 0 points }} \\
\hline & Alert & & \\
\hline & Arousal by stimulation & \multicolumn{2}{|l|}{1 point } \\
\hline & No response & \multicolumn{2}{|l|}{2 points } \\
\hline \multirow{3}{*}{$\begin{array}{l}\text { Consciousness } \\
\text { disturbance }\end{array}$} & Ask for patient's name & \\
\hline & Correct & Normal: 0 points & \\
\hline & Incorrect & 1 point & \\
\hline \multirow{8}{*}{$\begin{array}{l}\text { Motor weakness } \\
\text { (kinetic paralysis) }\end{array}$} & $\begin{array}{l}\text { Ask the patient to close eyes, raise arms to the front with palms down } \\
\text { (with gestures) }\end{array}$ & Right hand & Left hand \\
\hline & Being able to keep arms raised in parallel & Normal: 0 points & Normal: 0 points \\
\hline & Being able to raise arms but not to keep them in position so that they go downward & 1 point & 1 point \\
\hline & Unable to raise arms & 2 points & 2 points \\
\hline & Instruct the patient to close eyes, raise legs from the bed to the front with gestures & Right leg & Left leg \\
\hline & Being able to keep legs raised in parallel & Normal: 0 points & Normal: 0 points \\
\hline & Being able to raise legs but not to keep them in position so that they go downward & 1 point & 1 point \\
\hline & Unable to raise legs & 2 points & 2 points \\
\hline \multirow{4}{*}{ Language } & Ask the patient to say 'It's fine today' repeatedly & & \\
\hline & Being able to clearly say it repeatedly & \multicolumn{2}{|l|}{ Normal: 0 points } \\
\hline & Either unclear (slurred) or abnormal & \multicolumn{2}{|l|}{1 point } \\
\hline & Keeping silent, unable to understand linguistically & \multicolumn{2}{|l|}{2 points } \\
\hline \multicolumn{2}{|l|}{ Total } & \multicolumn{2}{|l|}{...points } \\
\hline
\end{tabular}

Fig. 3. Kurashiki Prehospital Stroke Scale. Adapted from Iguchi, et al. Cerebrovase Dis 2011;31:51-6 with permission from S. Karger AG. Basel. ${ }^{3}$

Although IV-tPA was administered to acute stroke patients within 3 hours of onset, the patients whose condition had not improved at the end of IV-tPA infusion were subsequently treated with selective IA-UK. The strategy of using conventional-dose IV-tPA followed by IA-UK in patients without an early clinical response to IV treatment was shown to be both safe and feasible. This strategy achieved high rates of complete arterial recanalization and good functional outcomes. ${ }^{12,13}$ Considering the benefits of treating acute ischemic stroke, EMS personnel should consider triaging to a stroke center, which is best able to manage the patient, by assessing stroke severity within an appropriate period of time. ${ }^{2}$ The widely used CPSS and LAPSS can be used to rapidly recognize acute stroke, but these prehospital stroke scales cannot determine the severity of a stroke. ${ }^{3}$

It is difficult to apply the NIHSS to stroke patients on the scene. ${ }^{8}$ However, our results showed that the KPSS strongly correlates with the NIHSS in patients both within 3 hours and 6 hours of stroke onset. Unlike previous studies with a cutoff KPSS of 4, a patient with a KPSS score $\geq 3$ can be considered for administration of both IV-tPA and IA-UK when symptom onset was within 3 and 6 hours. A cut-off KPSS value of 3 was also estimated for patients actually receiving IV-tPA and IA-UK. A prehospital stroke scale for assessing severity should be pursued as a screening tool for patients requiring crucial treatments. Compared to previous studies showing a $69 \%$ sensitivity and $63 \%$ specificity at a cut-off of 4 for the administration of IV-tPA, these results demonstrate that a cut-off of 3 could improve both sensitivity and specificity and can be applied to stroke patients within the 6 hour symptom onset window. On scene, the KPSS can be used to identify candidates for IV-tPA and IA-UK, evidenced by its excellent correlation with the NIHSS in the assessment of stroke severity. Additionally, KPSS can be used to more rapidly initiate stroke management by faster triage of patients in the hospital.

\section{Limitations}

There are several limitations to our study. First, although the KPSS is a useful prehospital stroke scale, our KPSS assessments were not determined by EMTs on scene, but were instead conveniently determined by EMTs in the ED. Second, the BEST program was only activated if at least one of five stroke warning signs described by the AHA was identified within 12 hours after symptom onset. After initial screening by BEST activation, the patient's KPSS score could be assessed by an EMT. There might be selection bias. Third, although an educational program for the prehospital stroke scale, including KPSS, was provided to participants, inter-observer reliability could not be established 
for KPSS among EMTs or for NIHSS among neurologists in our study. There is also a discrepancy in the timing of the KPSS and NIHSS assessments. Any difference in symptoms could not be accounted for in our study. Fourth, new evidence for IA-UK in patients with vertebral or basilar artery occlusion within 24 hours of symptoms onset suggests that intra-arterial thrombolysis may be used. ${ }^{3}$ Our study was limited to patients presenting within 6 hours of symptom onset. In the future, a larger prospective study with KPSS assessment performed by EMTs on the scene within 24 hours of symptom onset will be needed to investigate the effectiveness of this stroke triaging tool.

In conclusion, the KPSS is an effective prehospital stroke scale for the assessment of stroke severity that can be used to identify candidates for IV-tPA and IA-UK as evidenced by excellent correlation with the NIHSS in patients with acute ischemic stroke.

\section{ACKNOWLEDGEMENTS}

This research was supported by Basic Science Research Program through the National Research Foundation of Korea (NRF) funded by the Ministry of Education (NRF2013R1A1A2009391).

\section{REFERENCES}

1. Jung S, Kwon SB, Hwang SH, Noh JW, Lee YK. Ischemic stroke among the patients with end-stage renal disease who were undergoing maintenance dialysis. Yonsei Med J 2012;53:894-900.

2. Jauch EC, Cucchiara B, Adeoye O, Meurer W, Brice J, Chan YY, et al. Part 11: adult stroke: 2010 American Heart Association Guidelines for Cardiopulmonary Resuscitation and Emergency Cardiovascular Care. Circulation 2010;122(18 Suppl 3):S818-28.
3. Iguchi Y, Kimura K, Watanabe M, Shibazaki K, Aoki J. Utility of the Kurashiki Prehospital Stroke Scale for hyperacute stroke. Cerebrovasc Dis 2011;31:51-6.

4. Adams HP Jr, del Zoppo G, Alberts MJ, Bhatt DL, Brass L, Furlan A, et al. Guidelines for the early management of adults with ischemic stroke: a guideline from the American Heart Association/ American Stroke Association Stroke Council, Clinical Cardiology Council, Cardiovascular Radiology and Intervention Council, and the Atherosclerotic Peripheral Vascular Disease and Quality of Care Outcomes in Research Interdisciplinary Working Groups: the American Academy of Neurology affirms the value of this guideline as an educational tool for neurologists. Stroke 2007;38: 1655-711.

5. Kothari RU, Pancioli A, Liu T, Brott T, Broderick J. Cincinnati Prehospital Stroke Scale: reproducibility and validity. Ann Emerg Med 1999;33:373-8.

6. Kidwell CS, Starkman S, Eckstein M, Weems K, Saver JL. Identifying stroke in the field. Prospective validation of the Los Angeles prehospital stroke screen (LAPSS). Stroke 2000;31:71-6.

7. Nam HS, Han SW, Ahn SH, Lee JY, Choi HY, Park IC, et al. Improved time intervals by implementation of computerized physician order entry-based stroke team approach. Cerebrovasc Dis 2007;23:289-93.

8. Llanes JN, Kidwell CS, Starkman S, Leary MC, Eckstein M, Saver JL. The Los Angeles Motor Scale (LAMS): a new measure to characterize stroke severity in the field. Prehosp Emerg Care 2004;8:46-50.

9. Kimura K, Inoue T, Iguchi Y, Shibazaki K. Kurashiki prehospital stroke scale. Cerebrovasc Dis 2008;25:189-91.

10. Heo JH, Kim YD, Nam HS, Hong KS, Ahn SH, Cho HJ, et al. A computerized in-hospital alert system for thrombolysis in acute stroke. Stroke 2010;41:1978-83.

11. Ogawa A, Mori E, Minematsu K, Taki W, Takahashi A, Nemoto S, et al. Randomized trial of intraarterial infusion of urokinase within 6 hours of middle cerebral artery stroke: the middle cerebral artery embolism local fibrinolytic intervention trial (MELT) Japan. Stroke 2007;38:2633-9.

12. Lee KY, Kim DI, Kim SH, Lee SI, Chung HW, Shim YW, et al. Sequential combination of intravenous recombinant tissue plasminogen activator and intra-arterial urokinase in acute ischemic stroke. AJNR Am J Neuroradiol 2004;25:1470-5.

13. IMS Study Investigators. Combined intravenous and intra-arterial recanalization for acute ischemic stroke: the Interventional Management of Stroke Study. Stroke 2004;35:904-11. 